\title{
Prekäre Wohnverhältnisse, Verdrängungsdruck und die Entstehung politischer Kollektivität in Frankfurt Westhausen
}

\author{
Falk Künstler • Sebastian Schipper
}

Eingegangen: 8. Juni 2021 / Angenommen: 13. Oktober 2021 / Online publiziert: 12. November 2021

(C) Der/die Autor(en) 2021

Zusammenfassung Auf Basis ethnographischer Feldforschung untersucht der Beitrag den Zusammenhang zwischen prekären Wohnverhältnissen und der Entstehung politischer Kollektivität unter Mieter*innen. Theoretisch-konzeptionell knüpft er dabei an Debatten der kritischen Stadtforschung zu Verdrängungsprozessen, Forschungsarbeiten zu Prekarisierung sowie poststrukturalistisch inspirierte Perspektiven der sozialen Bewegungsforschung an. Empirisch ist der Blick gerichtet auf die Lebensrealitäten von Bewohner*innen einer Frankfurter Vonovia-Wohnsiedlung. Gefragt wird danach, wie Mieter*innen ihre Wohnsituation in Anbetracht drohender Mieterhöhungen infolge von Modernisierungsarbeiten wahrnehmen, welche Bedeutung sie der Wohnungsfrage zuschreiben und welche sozialräumlichen Bedingungen, Handlungspraktiken und diskursiven Deutungsmuster die Entwicklung einer kollektiven politischen Handlungsfähigkeit erschweren.

Schlüsselwörter Wohnen · Verdrängung · Prekarität · Politische Kollektivität · Städtische soziale Bewegungen

Falk Künstler $(\bowtie) \cdot$ Sebastian Schipper

Institut für Humangeographie, Goethe-Universität Frankfurt am Main,

Theodor-W.-Adorno-Platz 6, 60629 Frankfurt am Main, Deutschland

E-Mail: f.kuenstler@em.uni-frankfurt.de

Sebastian Schipper

E-Mail: S.Schipper@geo.uni-frankfurt.de 


\title{
Precarious housing conditions, displacement pressure and emergence of political collectivity in Frankfurt Westhausen
}

\begin{abstract}
Based on ethnographic fieldwork, this paper analyzes the nexus between precarious housing conditions and the emergence of political collectivity among tenants. Our theoretical framework brings together literature on displacement discussed in critical urban studies, debates on precarity and social insecurity as well as poststructuralist perspectives in social movement research. Empirically our view is centered on a privatized housing estate in Frankfurt Westhausen owned by Germany's largest financialized housing company Vonovia. We investigate how tenants perceive their housing situation in the face of impending rent increases as a result of modernization work, what meaning they ascribe to the housing situation, and what sociospatial conditions, practices, and discourses impede the development of political collectivity.
\end{abstract}

Keywords Housing · Displacement · Precarity · Political collectivity · Urban social movements

\section{Einleitung}

Die wohnungspolitische Situation speziell von einkommensärmeren Haushalten in deutschen Großstädten lässt sich gegenwärtig wie folgt umreißen: Während die Nachfrage nach bezahlbarem Wohnraum das Angebot bei Weitem übersteigt, findet ein Großteil des Wohnungsneubaus im gehobenen Preissegment statt und wird in Form von Eigentumswohnungen vermarktet. Bedingt durch Prozesse der Privatisierung und Finanzialisierung der Wohnungsversorgung führen zugleich rasante Aufwertungsdynamiken auch im Bestand zu explodierenden Mietpreisen, was vor allem in innerstädtischen Gebieten mit Verdrängungsprozessen einhergeht (Beran und Nuissl 2019; Helbrecht 2016; Mösgen und Schipper 2017). Verstärkt wird diese Entwicklung durch den Rückzug des Staates aus dem sozialen Wohnungsbau. Auslaufende Bindungen und ausbleibender Neubau haben den Bestand an Sozialwohnungen von über 4Mio. Ende der 1980er Jahre auf gut 1 Mio. gebundene Wohneinheiten heutzutage abschmelzen lassen (Schönig 2020). Zugleich sehen sich untere Einkommensgruppen oft zusätzlich den Implikationen neoliberalisierter Arbeitsmärkte und Rentensysteme ausgesetzt (Nachtwey 2016, S. 120 ff.). In der gegenwärtigen Konstellation, in der prekäre Beschäftigungsverhältnisse, sinkende oder stagnierende Realeinkommen und privatisierte Risiken auf einen Mangel an bezahlbarem Wohnraum und damit einhergehend auf fehlende Umzugsalternativen treffen, stellen Mieterhöhungen oder gar die Aufkündung des bestehenden Mietverhältnisses für viele Menschen eine existenzielle Bedrohung dar. In der Stadtforschung werden diese Entwicklungen seit einigen Jahren als erneute Rückkehr der „Wohnungsfrage“ diskutiert (Baldenius et al. 2020; Belina 2018; Egner et al. 2021; Rink et al. 2015; Schönig und Vollmer 2020).

Angesichts der hier knapp skizzierten wohnungspolitischen Transformationsprozesse, der Zunahme von Wohnungsnot und der existenziellen Bedrohung, die der 
Verlust der eigenen Wohnung für viele Haushalte bedeutet, ist in jüngerer Zeit und ausgehend von zahlreichen lokalen Initiativen eine neue mietenpolitische Bewegung entstanden, die gegen die Vermarktlichung und Privatisierung der Wohnungsversorgung aufbegehrt, Verdrängungsprozesse skandalisiert und relativ erfolgreich für einen grundlegenden Wandel der Wohnungspolitik mobilisiert (Rink und Vollmer 2019; Schipper 2018; Vogelpohl et al. 2017). Bestehende mietenpolitische Initiativen rekrutieren sich allerdings meist überwiegend aus Mieter*innen der Mittelschicht. Gerade marginalisierte Bevölkerungsgruppen, für welche die aktuelle wohnungspolitische Lage häufig existenzgefährdend ist, scheinen selten aktiv zu partizipieren bzw. kollektiv Widerstand gegen ihre Verdrängung zu organisieren (Ehlers 2021). In diesem Punkt unterscheidet sich der jüngste Protestzyklus nicht wesentlich von früheren Phasen stadtpolitischer Gegenwehr. Kerstin Dörhöfer (1978, S. 80) diagnostizierte bereits in Hinblick auf wohnungspolitische Proteste der 1970er Jahre, dass „Initiatoren und Träger der Aktionen selten jene sind, die von existenzieller oder gewohnter" sozialer Not betroffen sind, also die Bevölkerungsgruppen mit niedrigstem Einkommen: Sozialhilfeempfänger, Arbeitslose, ungelernte Arbeitskräfte, Hausfrauen, Rentner. Widerstand und Proteste gegen Mietpreiserhöhungen, unterlassene Instandsetzungen, drohenden Abriß, fehlende Wohnfolgeeinrichtungen kommen meist von Angehörigen des Mittelstandes“.

In Anbetracht dieser Diagnose ist das Erkenntnisinteresse der Untersuchung auf die Frage gerichtet, warum Mieter*innen in prekären Wohnverhältnissen nur selten kollektiv Widerstand gegen ihre drohende Verdrängung und die Verschlechterung ihrer Lebensverhältnisse leisten bzw. welche sozialräumlichen Bedingungen, Handlungspraktiken und diskursiven Deutungsmuster die Entwicklung einer gemeinsamen politischen Kollektivität erschweren. Auf Basis ethnographischer Feldforschung haben wir dazu untersucht, wie untere Einkommensschichten ihre Wohnsituation angesichts drohender Mieterhöhungen und einer potenziellen Verdrängung wahrnehmen, welche Bedeutung sie der Wohnungsfrage zuschreiben und inwiefern eine kollektive politische Handlungsfähigkeit entsteht. Im Sinne einer „Angewandten Kritischen Geographie“ (Kuge et al. 2020) greift die Studie damit auch ein Problem der politischen Praxis wohnungspolitischer Initiativen auf, indem sie Antworten auf die Frage bietet, wie es gelingen kann, dass auch marginalisierte Gruppen, die am unmittelbarsten von Wohnungsnot, Verdrängung und steigenden Mieten betroffen sind, ihre Interessen kollektiv artikulieren und - sicherlich nicht zwingend (Steenblock und Petzold 2021), aber im besten Fall (Ehlers 2021; Vollmer 2019) - darüber letztlich Einfluss auf wohnungspolitische Entscheidungsprozesse gewinnen.

Als empirisches Fallbeispiel dient uns die Siedlung Westhausen in Frankfurt am Main, in welcher derzeit 260 Haushalte von Modernisierungsmieterhöhungen betroffenen sind. Innerhalb der Wohnsiedlung - deren größter Teil sich in der Hand der öffentlichen Wohnungsgesellschaften ABG Frankfurt Holding und Nassauische Heimstätte befindet - wurden diese 260 Wohneinheiten 2002 an die Viterra AG privatisiert und gehören nach der Übernahme durch die Deutsche Annington 2005 heute dem größten deutschen Immobilienkonzern Vonovia SE (Unger 2018). Im Gegensatz zum älteren Siedlungsteil aus der Ära des Frankfurter Städtebauprogramms ,Neues Frankfurt` der 1920er Jahre wurden die hier betrachteten Wohneinheiten in den 
1950er Jahren als vierstöckige Mehrfamilienhäuser in Plattenbauweise errichtet. ${ }^{1}$ Nach Jahren vernachlässigter Instandhaltung durch den Wohnungskonzern ist der bauliche Zustand der Häuser zum Zeitpunkt der Feldforschung äußerst schlecht dazu gehören beispielsweise nasse Keller, bröckelnde Fassaden, rostige Geländer, kaputte Briefkästen oder Sprechanlagen. Ende 2018 werden die Mieter*innen benachrichtigt, dass umfangreiche Baumaßnahmen stattfinden sollen. Dabei handelt es sich allerdings nicht um die seit Jahren von Bewohner*innen eingeforderten Instandhaltungen, welche von dem Wohnungsunternehmen selbst zu finanzieren wären, sondern um Modernisierungen, deren Kosten von den Mieter*innen getragen werden müssen. Diese beinhalten den Austausch von Fenstern und Eingangstüren, Wärmedämmung oder größere Balkone. Die Kosten können nach Abschluss der Arbeiten dauerhaft auf die Mieter*innen umgelegt werden, woraus monatliche Mieterhöhungen von circa 60 bis $120 €$ pro Wohneinheit resultieren. ${ }^{2}$ Um die angekündigten Maßnahmen zu diskutieren, trifft sich auf Initiative eines in der Siedlung tätigen Sozialarbeiters sowie der Mitarbeiterin eines Frankfurter Mietervereins im Dezember 2018 erstmalig eine Gruppe von Bewohner*innen. Dieses Treffen bildet den Startpunkt der Mieterinitiative Westhausen, welche im Kern 15 bis 20 Personen umfasst und die bis heute regelmäßig in den Räumen des Jugendzentrums zusammenkommt, sofern dies die Corona-Pandemie zulässt. Ausgehend von dem Erkenntnisinteresse, den Zusammenhang zwischen prekären Wohnverhältnissen und (ausbleibenden) Prozessen einer politischen Kollektivität zu beleuchten, wurde im April 2019 eine bis Januar 2020 andauernde ethnographische Feldforschung begonnen, die im Kern auf einer teilnehmenden Beobachtung und Protokollierung der monatlichen Treffen der Initiative, fünf leitfadengestützten Interviews, kürzeren Gesprächen mit weiteren Bewohner*innen sowie Beobachtungen im öffentlichen Raum der Siedlung beruht. ${ }^{3}$

Im folgenden Abschn. 2 werden wir zunächst die Untersuchung theoretisch-konzeptionell in die Stadt-, Prekarisierungs- und soziale Bewegungsforschung einbetten und die zentralen Begriffe Verdrängung, Prekarisierung und politische Kollektivität diskutieren. Daran anschließend werden wir auf Basis des empirischen Materials in Abschn. 3 zeigen, dass erstens die sozioökonomische Heterogenität inner-

\footnotetext{
${ }^{1}$ Die Zweiteilung der Siedlung drückt sich auch im aktuellen Mietspiegel aus. Der untersuchte Siedlungsteil aus den 1950er-Jahren gilt demnach als „einfache Wohnlage“, die ältere Siedlungshälfte des Neuen Frankfurts hingegen als höherwertige „,mittlere Wohnlage“ (Amt für Wohnungswesen Stadt Frankfurt am Main 2018, S. 22).

${ }^{2}$ Laut Aussage der interviewten Mieter*innen lag die Warmmiete für die überwiegend 55 bis $70 \mathrm{~m}^{2}$ großen Wohnungen vor der Modernisierung zwischen 660 und $800 €$.

3 Die Leitfadeninterviews setzen sich aus zwei Einzelinterviews und einem Doppelinterview mit Mieter*innen der Mieterinitiative Westhausen sowie zwei Expertengesprächen mit einem vor Ort tätigen Sozialarbeiter und einer Mitarbeiterin eines lokalen Mietervereins zusammen. Diese fünf ein- bis zweistündigen Interviews wurden transkribiert und in Anlehnung an Strategien der Grounded Theory ausgewertet. Die jeweils verwendeten Leitfäden wurden entsprechend dem aktuellen Erkenntnisstand der Studie angepasst, jedoch offen gestaltet, um narrative Phasen anzustoßen und den Befragten ausreichend Raum für eigene Deutungen, Assoziationen und Schwerpunktsetzungen zu bieten. Zusätzlich gingen aus der teilnehmenden Beobachtung im Rahmen der Treffen der Mieterinitiative, aus Aufenthalten vor Ort sowie spontanen Gesprächen mit weiteren Mieter*innen Beobachtungsprotokolle und Feldnotizen hervor, die wie die Interviewtrankskripte ebenfalls durch das Schreiben von Memos begleitet sowie kodiert wurden. Die Auswahl der Gesprächspartner*innen war im Sinne eines Theoretical Samplings vom jeweiligen Erkenntnisstand geleitet.
} 
halb der Siedlung, Sprachbarrieren sowie schwach ausgebildete soziale Netzwerke als sozialräumliche Bedingungen die Entstehung einer politischen Kollektivität erschweren. Auf Ebene der Handlungspraktiken wirken zweitens insbesondere bei einkommensärmeren Mieter*innen zudem Zukunftsängste, Ohnmachtsgefühle und eine pessimistische Wahrnehmung der eigenen Handlungsmacht kollektiven Politisierungsprozessen entgegen. Schließlich kann drittens auch die Analyse verbreiteter Deutungsmuster verstehbar machen, warum sich unter den Mieter*innen nicht mehr Widerstand gegen die Modernisierung und die damit einhergehenden Mieterhöhungen regt. Beispielsweise erzeugt die territoriale Stigmatisierung (Wacquant 2008) der Siedlung bei manchen Bewohner*innen Scham. Infolgedessen werden die Modernisierungsmaßnahmen auch von Mieter*innen in prekären Wohnverhältnissen, die bereits mit einer hohen Mietbelastung konfrontiert sind, mit der Hoffnung verbunden, durch die Aufwertung vom Stigma des schlechten Wohnviertels befreit zu werden. Über mehrere Monate hinweg erweist sich die Mieterinitiative allerdings in ihrer Ausrichtung als bemerkenswert dynamisch und bringt trotz der herausgearbeiteten Hemmnisse zunehmend kollektive und politische Handlungspraktiken hervor. Auf den empirischen Erkenntnissen aufbauend, argumentieren wir abschließend, dass der Wille, Probleme der Wohnsituation kollektiv und politisch adressieren zu wollen, sich weder automatisch aus der vermeintlich objektiven gemeinsamen Interessenslage noch aus einer a priori feststehenden und geteilten Identität ableiten lässt, sondern erst aus vergemeinschafteten Erfahrungen und Praktiken heraus erwachsen kann. Das Fallbeispiel zeigt dabei auch, dass externe Akteure bzw. Fachpersonal der Sozialen Arbeit durch entscheidende Impulse und eine langfristig angelegte Unterstützung die Entstehung einer politischen Kollektivität wesentlich begünstigen können (Abschn. 4).

\section{Verdrängung, Prekarisierung und politische Kollektivität}

Die vorliegende Untersuchung nimmt Bezug auf Debatten der kritischen Stadtforschung zu Verdrängungsprozessen (2.1), auf Forschungsarbeiten zu Prekarisierung (2.2) sowie auf poststrukturalistisch inspirierte Perspektiven der sozialen Bewegungsforschung zur Entstehung politischer Kollektivität (2.3).

\subsection{Gentrifizierungsforschung und Verdrängung}

Im Kontext von Gentrifizierungsprozessen beschäftigt sich die Stadtforschung überwiegend mit der Aufwertung von Quartieren sowie deren Ursachen und Verlaufsformen (Lees et al. 2008; Üblacker 2018). Nur wenige empirische Untersuchungen nehmen die Betroffenen der Gentrifizierung selbst, nämlich die verdrängten oder von Verdrängung bedrohten Haushalte, in den Blick. Dementsprechend betont etwa Ilse Helbrecht (2016, S. 10), dass durch die Fokussierung auf Phänomene der Aufwertung und Ursachengefüge „,die Folgen der Gentrification für die Verdrängten“ insbesondere in Studien seit den 1990er Jahren ,nahezu vollständig aus dem Blick“ geraten seien (ähnlich auch Slater 2006). Die relativ wenigen Arbeiten, die sich mit Verdrängungsprozessen beschäftigen, entstehen zudem meist in der Absicht, 
quantitativ die Zahl der von Verdrängung Betroffenen zu bestimmen (Beran und Nuissl 2019). Forschungsarbeiten, die sich explizit mit der Lebenswirklichkeit und den Bleibestrategien von einkommensarmen Gruppen beschäftigen, sind dagegen selten (Atkinson 2015; Bernien 2017; Meuth und Reutlinger 2021; Reutlinger et al. 2019; Valli 2015; Winke 2020). Ein wesentlicher Grund für den Mangel an Arbeiten über von Verdrängung bedrohte Haushalte liegt in dem forschungspraktischen Problem begründet, dass einkommensarme Haushalte in einem gentrifizierten Stadtteil zum Zeitpunkt des Untersuchungsbeginns oft bereits verdrängt worden sind, folglich nicht mehr im Untersuchungsgebiet wohnen und dort auch nicht mehr befragt werden können. ${ }^{4}$ Als Konsequenz dieser Überlegung haben wir eine Siedlung in den Blick genommen, in der zu Beginn der Feldforschung die Aufwertung des Wohnungsbestandes noch am Anfang stand und wo damit auch mögliche Verdrängungsprozesse noch nicht stattgefunden hatten.

\subsection{Prekarisierung und politische Partizipation}

Prekarisierung wird in der Literatur als übergreifendes Phänomen der Unsicherheit bzw. Verunsicherung diskutiert, welches sich in verschiedenste gesellschaftliche Bereiche hinein erstreckt. Bedingt durch unstetige oder schlecht entlohnte Beschäftigungsverhältnisse und eine reduzierte sozialstaatliche Absicherung drückt sich in der Prekarisierung die beständige Gefahr des sozialen Abstiegs aus (Marchart 2014; Nachtwey 2016). In Analogie dazu sind prekäre Wohnverhältnisse, die hier im Zentrum stehen, gleichermaßen von Unsicherheit geprägt. Konkret manifestiert sich eine Prekarisierung des Wohnens etwa in einer hohen und kaum noch tragbaren Mietbelastung, einer Überbelegung der Wohnung, gravierenden baulichen Mängeln oder dem drohenden Verlust des eigenen Wohnraums. Nach Marchart (2014, S. 18) kann Prekarität dabei nicht generell mit „Armut, Verelendung und Exklusion“ gleichgesetzt werden, da bestimmte Dimensionen der Unsicherheit durchaus auch Haushalte der Mittelschicht betreffen können - so teilweise auch bildungsstarke Milieus. Im Kontext der Wohnungsfrage etwa erfahren sowohl untere als auch zunehmend mittlere Einkommensgruppen die oben skizzierten Prekarisierungstendenzen.

Sozialstrukturanalytische Forschungsbeiträge zeigen, dass prekär lebende Menschen häufig von sozialer Desintegration betroffen sind, von partizipativen Prozessen nur selten erreicht werden, vielfach resignieren und sich politisch kaum organisieren (Böhnke 2011; Nachtwey 2016). Candeias (2009, S. 369) hebt jedoch kritisch hervor, dass Personen in prekären Lebenssituationen oftmals vorschnell soziale Vereinzelung und Unorganisierbarkeit bescheinigt und selbige zu wenig als handelnde Subjekte ernst genommen würden. Das Leben von Menschen in prekären Arbeitsund Wohnverhältnissen wäre zwar in der Tat in erster Linie von Zwängen sowie von gesellschaftlicher Exklusion bestimmt; Phänomene der Selbstorganisation würden

\footnotetext{
4 Die unter anderem damit verbundenen konzeptionellen und methodisch-methodologischen Herausforderungen haben jüngst Miriam Meuth und Christian Reutlinger (2021) anhand empirischer Feldforschungen zu direkten Verdrängungsprozessen durch Totalsanierungen bzw. Ersatzneubauten in der Schweiz reflektiert, wodurch sie verschiedene Lösungswege für eine qualitativ ausgerichtete Verdrängungsforschung aufzeigen.
} 
allerdings zu voreilig ausgeblendet. Dennoch erscheint es plausibel und empirisch belastbar, davon auszugehen, dass „Prekarisierung und Ausgrenzung die Fähigkeit zu Widerstand und Protest schwächen“" (Dörre 2010, S. 231). Dieser Befund sagt aber als solcher wenig darüber aus, was prekäre Wohnverhältnisse oder eine akut bedrohte Wohnsituation unter Betroffenen tatsächlich auslösen und welche Handlungspraktiken und Denkmuster sie anstoßen, festigen oder auch aufbrechen. Deshalb wird diese Studie am Fallbeispiel Westhausen empirisch erschließen, wie sich (fehlende) Teilhabe und (ausbleibende) politische Kollektivität unter Bedingungen prekärer Wohnverhältnisse darstellen und was wiederum die Gründe dafür sind.

\subsection{Soziale Bewegungsforschung und politische Kollektivität}

Dem Beitrag liegt die zentrale Frage zugrunde, weshalb es nicht öfter zu kollektivem und politischem Widerstand derjenigen kommt, die besonders von prekären Wohnverhältnissen und Verdrängungsprozessen betroffen sind. Der Frage nach der Entstehung politischer Kollektivität widmen sich insbesondere marxistische Klassentheorien, aber auch poststrukturalistische Forschungsansätze zur Entstehung neuer sozialer Bewegungen. Klassentheorien folgend resultiert politische Kollektivität aus einem objektiv gegebenen Interesse von Menschen in gleicher Klassenlage, welche sich wiederum aus der Position innerhalb der herrschenden Produktions- und Eigentumsverhältnisse und insbesondere aus der Stellung im Produktionsprozess und der Verfügung über Produktionsmittel ergibt. Inwiefern allerdings eine eindeutige Klassenzuordnung und - darin begründet - die Formierung politischer und kollektiver Subjekte innerhalb einer fragmentierten und individualisierten Gesellschaft möglich ist, wird auch in marxistischen Debatten kontrovers diskutiert (Thien 2014). Entsprechend wird auch mit Bezug zur Wohnungsfrage problematisiert, dass die gemeinsame Betroffenheit von prekären Wohnverhältnissen keine materielle Ausgangsbasis begründet, die bereits als solche eine gemeinsame politische Interessenskonstellation hervorbringt. Betont wird vielmehr, dass die Eigenschaft, zur Miete zu wohnen, erst einmal keine stabile Grundlage für eine politische Kollektivität bildet, und zudem Klassenstruktur und soziale Zusammensetzung in städtischen Nachbarschaften oft heterogen sind (Vollmer 2019, S. 15 ff.).

Vor diesem Hintergrund richtet die poststrukturalistisch inspirierte soziale Bewegungsforschung zu wohnungspolitischen Protesten ihren Fokus stärker auf die Subjekte selbst und von dort ausgehend auf den Prozess der Konstruktion gemeinsamer Identitäten (Hölzl 2015; Rutland 2013; Scheller 2019; Vollmer 2019). Gefragt wird dabei nach den konkreten Praktiken, die geteilte Interessen hervorbringen und gemeinsame Identitäten konstruieren und erst so ein politisches Subjekt entstehen lassen. Letzteres kann dann auch quer zu sozialstrukturellen Trennlinien wie Klasse, Ethnizität, Religion etc. verlaufen. Insbesondere Lisa Vollmer (2019) hat kürzlich wichtige Erkenntnisse vorgelegt, die das Verständnis aktueller Formierungsprozesse von wohnungspolitischen Initiativen schärfen. Ausgehend von der postfundamentalistischen Sozialtheorie von Ernesto Laclau und am Beispiel mietenpolitischer Bewegungen in Berlin und New York arbeitet sie heraus, dass der Formierung politischer Kollektivität keine geteilten Interessen und Identitäten vorausgehen. Vielmehr bilden sich diese erst durch alltägliche Praktiken, welche hegemoniale Ordnungen 
aufbrechen, neue Deutungsangebote schaffen und einen universellen und verbindenden Horizont gemeinsamer Forderungen aufspannen. Dabei wirkt eine akute Bedrohung der eigenen Wohnsituation häufig als entscheidende Dislokation gewohnter Handlungspraktiken und bestehender gesellschaftlicher Arrangements. Dieser Bruch schafft die notwendige Bedingung, dass politische Subjekte als potenzielle „Träger von Veränderungen“ (Vollmer 2019, S. 31) hervortreten. Politische Kollektivität entsteht allerdings erst dann, wenn einzelne Subjekte von ihren individuellen Interessen und Problemen abstrahieren und Erfahrungen innerhalb einer Gruppe von Menschen „vergemeinschaftet“ (Vollmer 2019, S. 125) werden können.

Meist richtet die städtische soziale Bewegungsforschung ihren Fokus auf relativ erfolgreiche Initiativen, denen die Formierung einer politischen Kollektivität gelungen ist und denen breite öffentliche Aufmerksamkeit zuteilwird (Mullis 2017; Schipper 2017; Vollmer 2019). Der vorliegende Beitrag verschiebt diesbezüglich leicht die Perspektive, indem er sich einer Initiative zuwendet, bei der verschiedene Hemmnisse den Prozess der Entstehung einer politischen Kollektivität deutlich erschweren. Dadurch können wesentliche Bedingungen und Mechanismen sichtbar gemacht werden, die kollektive und politische Formen des Protests und Widerstands unter Mieter*innen in prekären Wohnverhältnissen blockieren und welche auch über das konkrete Fallbeispiel hinaus in anderen Kontexten Relevanz entfalten.

\section{Zur Entstehung politischer Kollektivität unter Mieter*innen in Frankfurt-Westhausen}

Die Analyse des empirischen Materials entlang der Fragen, wie die Mieter*innen in Frankfurt-Westhausen ihre individuelle Wohnsituation wahrnehmen, wie sie die Modernisierungsarbeiten und drohenden Mieterhöhungen bewerten und wie sie ihre Handlungsmöglichkeiten einschätzen, hat vier zentrale Ebenen zum Vorschein gebracht. Zum einen wirkt die sozialstrukturelle Heterogenität der Siedlung und die damit verbundenen Ausschlussmechanismen als sozialräumliche Bedingungen der Ausbildung einer politischen Kollektivität entgegen (3.1). Zweitens erzeugen auf Ebene der Handlungspraktiken individuelle Strategien, Zukunftsängste und Ohnmachtsgefühle gleichermaßen eine hemmende Wirkung auf Prozesse der kollektiven Politisierung (3.2). Schließlich erschweren drittens ebenso verbreitete Deutungsmuster die Entwicklung solidarischer Perspektiven (3.3). Entgegen dieser drei Hemmnisse sind es die konkreten Praktiken der Vergemeinschaftung von Erfahrungen und Problemen durch die Mieterinitiative selbst, welche im zeitlichen Verlauf auf Subjektebene Veränderungen erzeugen und die Entstehung einer politischen Kollektivität zumindest in Ansätzen möglich machen (3.4).

\subsection{Sozialräumliche Bedingungen: Heterogenität und Barrieren}

Erschwert wird die Entstehung einer politischen Kollektivität in Westhausen auf sozialräumlicher Ebene durch die sozioökonomischen Gegensätze innerhalb der Siedlung und dabei vor allem durch die Akzeptanz der Modernisierungsmieterhöhungen seitens der ökonomisch bessergestellten Haushalte. Sozialstrukturelle Faktoren bzw. 
die individuell unterschiedlichen Ressourcenausstattungen der Mieter*innen nehmen gewichtigen Einfluss auf die Beurteilung der aktuellen Wohnsituation sowie der anstehenden Modernisierungsarbeiten. Insbesondere ältere und langjährige Bewohner*innen mit deutscher Staatsbürgerschaft verfügen oft über sichere Gehälter oder als ehemalige Beamte über auskömmliche und stabile Pensionen. Gleichzeitig profitieren sie meist von alten und daher relativ günstigen Mietverträgen. Viele jüngere Haushalte sind hingegen erst in den letzten Jahren in die Siedlung gezogen, zahlen damit deutlich höhere Mieten und arbeiten häufiger in prekären Beschäftigungsverhältnissen. Zudem verfügen die relativ neuen Bewohner*innen mehrheitlich über Migrationserfahrungen. Die aktiven und regelmäßigen Mitglieder der Mieterinitiative lassen sich zu großen Teilen der ersten Gruppe zuordnen; sie sind meist über 65 Jahre alt, fast ausnahmslos deutschsprachig und ohne Migrationserfahrung und beziehen mehrheitlich Pensionen. Diese älteren Mieter*innen thematisieren im Rahmen der Initiative vornehmlich die unmittelbaren Einschränkungen durch die Bauarbeiten beispielsweise in Gestalt von Lärm und Dreck. Die Initiative entscheidet daher schon kurz nach ihrer Gründung mehrheitlich, die Modernisierungsmaßnahmen als solche zu akzeptieren und lediglich eine Reduktion individueller Einschränkungen durch die baulichen Maßnahmen zur Kernaufgabe der Gruppe zu machen. Die Mieterhöhung und deren Folgen sind für viele ältere Bewohner*innen dagegen nebensächlich. Kaum in die Mieterinitiative eingebunden werden diejenigen Haushalte, deren finanzielle Situation prekär ist und die sich daher unmittelbar von Verdrängung und einem möglichen Verlust der Wohnung bedroht sehen.

Als Gründe für diesen Ausschlussmechanismus sind Sprachbarrieren, Zeitmangel prekär Beschäftigter sowie schwach ausgebildete soziale Netzwerke zu nennen. Ohne dass die Siedlung Westhausen einen für Frankfurt außergewöhnlich hohen Anteil von Bewohner*innen mit Migrationserfahrung aufweisen würde, hat sich die Zahl migrantischer Haushalte in den letzten Jahren deutlich erhöht. Die damit verbundene große Sprachvielfalt sowie die mangelnden Fremdsprachenkenntnisse der älteren Mieterschaft haben Sprachbarrieren entstehen lassen, worunter die alltägliche Kommunikation in der Siedlung leidet und was die Entstehung sozialer Beziehungen sowie den Anschluss an die Mieterinitiative behindert:

selbst, wenn sie [...] zu unserem Treffen kämen, ja da ist wieder die sprachliche Barriere, wer übersetzt ihnen das, wer erklärt ihnen das genau? Das sind ja dann Stunden, wir sitzen ja da schon anderthalb, zwei Stunden, weil jeder halt was zu sagen hat und das erkläre mal bitte dann demjenigen, dann bist du in vier Stunden immer noch beschäftigt. Das ist so schwierig und wie gesagt, sie melden sich ja auch gar nicht. (Interview 5, Mieter*innen)

Die Möglichkeit der Teilnahme an der Initiative wird zudem durch vielfach prekäre Beschäftigungsformen (Schichtarbeit, Mehrfachbeschäftigung) erschwert. Darüber hinaus existieren städtebaulich in der isoliert gelegenen Siedlung nahezu keine gemeinschaftlich nutzbaren und öffentlich zugänglichen Orte. Neben dem Fehlen eines Gemeindetreffs oder einer einladenden Grünfläche sind ebenso frühere Dienstleistungsangebote (Reinigung, Arztpraxis), Kneipen oder kleine Geschäfte (Nahkauf, ,Tante-Emma-Laden“, Bäckerei) - und damit wichtige Begegnungsmöglichkeiten - in den letzten Jahrzehnten sukzessive verschwunden. Soziale Infrastruk- 
turen sind im Siedlungsleben mit Ausnahme des Jugendtreffs, welchen auch die Mieterinitiative auf Initiative des Sozialarbeiters nutzen kann, kaum noch existent:

Wie gesagt, kein Bürgertreff, [...] wo man sich einmal begegnen kann, wie soll man sich denn dann mit diesen neuen Leuten, die zugezogen sind, aus anderen

Kontinenten, wie soll man da mal auf jemanden zugehen, das ist ja auch schwer.

(Interview 3, Mieter)

Auf der Ebene der sozialräumlichen Bedingungen sind also zwei Phänomene fortlaufend präsent und einflussreich: Zum einen beeinflusst die heterogene Sozialstruktur der Siedlung die Perspektiven und prioritären Ansprüche der Bewohner*innen und erschwert dadurch die Entstehung einer politischen Kollektivität. Zum anderen werden der alltägliche Austausch und die soziale Vernetzung innerhalb der Siedlung als grundlegende Basis für die Entstehung einer politischen Kollektivität durch Sprachbarrieren, Zeitmangel und fehlende soziale Infrastrukturen und Begegnungsorte erheblich eingeschränkt. Die ressourcenschwächsten Haushalte sind zwar am stärksten von den Folgen der Modernisierung betroffen, aus den genannten Gründen jedoch kaum in die Struktur der Mieterinitiative eingebunden, wodurch sich ihre Positionen gegenüber der Vermietungsgesellschaft nur schwach artikuliert. Zudem entstehen durch diesen Ausschluss gravierende Informationsmängel, welche die Modernisierung und deren Konsequenzen, aber auch mögliche Hilfestellungen betreffen. Beispielsweise wird die Möglichkeit, bei hoher finanzieller Belastung Härtefallanträge gegenüber Vonovia zu stellen, nur von wenigen Haushalten wahrgenommen. Einer frist- und formgerechten Einreichung der Anträge stehen das notwendige Wissen um formelle Anforderungen sowie - noch grundlegender - die rechtzeitige Kenntnisnahme der äußerst knappen Fristsetzungen entgegen. Genau jene Haushalte, welche hierbei auf schnelle Unterstützung angewiesen wären, werden jedoch anfangs auch von existierenden Angeboten und Kompetenzen der Mieterinitiative oftmals nicht erreicht.

Zugleich macht das Fallbeispiel Westhausen aber ebenso deutlich, dass die sozialräumlichen Bedingungen (Heterogenität, Zeitmangel, Sprachbarrieren und fehlende soziale Begegnungsorte) durch das solidarische Engagement von externem Fachpersonal der Sozialen Arbeit und eines Mietervereins teilweise in ihrer Wirkung deutlich abgeschwächt werden können. Durch die anfängliche Übernahme von wesentlichen Organisationsaufgaben (z.B. Einladung zu sowie Moderation und Protokollierung der regelmäßigen Treffen, Aufbau von Kommunikationsstrukturen), die Vermittlung von ehrenamtlichen Übersetzungsdienstleistungen und die Bereitstellung von Räumen des Jugendzentrums als Anlaufpunkt für die Mieter*innen gelingt es den professionellen Akteuren, zuerst die Mieterinitiative ins Leben zu rufen, darauf aufbauend interne Prozesse der politischen Kollektivität anzustoßen und im weiteren Verlauf entscheidend zu deren Verstetigung und Fortentwicklung beizutragen (siehe 3.4).

\subsection{Konfliktvermeidung als dominante Handlungspraktik}

Manche Handlungspraktiken der Mieter*innen in Reaktion auf die Modernisierungsankündigung wirken auf den ersten Blick paradox. Darunter fällt die Beobachtung, 
dass Mieter*innen die umlagefähigen Modernisierungsmaßnahmen begrüßen und sich für eine kooperative Haltung gegenüber der Vermieterin aussprechen, obwohl der Wohnungskonzern seit Jahren die Forderungen von Mieter*innen nach dringend notwendigen Instandhaltungsarbeiten hartnäckig ignoriert. Widersprüchlich erscheint ebenso, dass manche Haushalte, die bereits vor Umlage der Modernisierungskosten mit einer hohen Mietbelastung konfrontiert waren ${ }^{5}$ und akut von Verdrängung bedroht sind, ebenso der Modernisierung zustimmen. Diese scheinbar paradoxen Handlungspraktiken der Mieter*innen lassen sich als Ausdruck der prekären Wohnverhältnisse verstehen.

\subsubsection{Individuelle statt konflikthafte Strategien}

Für die Mitarbeit in der Mieterinitiative zeigen sich anfangs als ausschlaggebende Motive überwiegend pragmatische Gründe, darunter vor allem die Kompensation der allgemein schlechten Informationslage bezüglich der Modernisierung. Politisch begründete Motive - beispielsweise eine Ablehnung mietpreissteigernder Aufwertungsmechanismen oder die Forderung nach solidarischem Handeln gegenüber finanziell überlasteten Nachbar*innen - sind hingegen kaum sichtbar. Gleichzeitig wird die zunehmende Bedrohung der Wohnsituation von vielen Mieter*innen lange Zeit als rein individuelles Problem wahrgenommen. Die unter den Bewohner*innen verbreitete Auffassung, die heterogene Ausgangslage der unterschiedlichen Haushalte würde verschiedene Bedürfnisse erzeugen und so einem gemeinsamen Vorgehen widersprechen, stützt diese Wahrnehmung. Die Gründung der Mieterinitiative bzw. die Beteiligung daran ist daher - zumindest in den Anfangsmonaten - mehrheitlich nicht Ausdruck eines Begehrens nach kollektivem politischem Widerstand, sondern dient vielen Mieter*innen als flankierendes Instrument der Informationsbeschaffung zur Stärkung individueller Aushandlungsstrategien. Einige Mieter*innen sprechen sich explizit dafür aus, mit dem zuständigen Bauleiter der Vonovia ,auf Vertrauensbasis [zu] kommunizieren“ (Beobachtungsprotokoll 12.06.2019). Mit partiellen und individuellen Strategien erzielen vor allem artikulationsstarke Mieter*innen kleinere Erfolge, wodurch sie anfangs in ihrem Vorgehen bestätigt werden. Kollektive Verhandlungen und konflikthafte Strategien erscheinen hingegen vergleichsweise unergiebig und wenig zielführend.

\subsubsection{Prekarisierung und Zukunftsängste: Die Wohnung um jeden Preis halten}

Die heterogene Sozialstruktur und die unterschiedlichen Ressourcen der Mieter*innen bringen ebenso unterschiedliche Wahrnehmungen der Wohnsituation hervor. Prekarität begründet sich gerade für einkommensärmere Mieter*innen aus dem Zusammenspiel einer bereits hohen Mietbelastungsquote, der angekündigten zusätzlichen Mietkosten und fehlender Alternativen an bezahlbarem Wohnraum im stadtweiten Kontext - schließlich liegen die Mietpreise der meisten Wohnungen

\footnotetext{
5 Ein gewichtiger Teil der Mieter*innen gab bereits vor den Modernisierungsarbeiten mehr als $30 \%$ ihres Einkommens für Mietzahlungen aus. Einige Haushalte lagen bei einer Mietbelastungsquote von $50 \%$ und mehr, gleichzeitig gab es aber auch einige Haushalte mit einer deutlich geringeren Belastung.
} 
auch nach der Modernisierung immer noch unter dem städtischen Durchschnitt der Angebotsmieten. Für einige dieser Mieter*innen erweist sich die Modernisierung als existenzielle Bedrohung ihres Mietverhältnisses. Hier werden Lebensmodelle sichtbar, in denen die Erwerbs- und Einnahmequellen passgenau auf die Lebenshaltungskosten abgestimmt sind, nun aber aufgrund der drastischen Mieterhöhungen zu zerfallen drohen.

Es sind viele Leute, die auch über staatliche Leistungen wie Aufstocken, Kindergeld, irgendwelche Zuschüsse sozusagen sich berappeln und ihr Leben irgendwie so hinbekommen, aber für die halt $100 €$ mehr Miete auf einmal das ganze System ins Ungleichgewicht bringt. (Interview 4, Mitarbeiterin Mieterverein)

Das Wissen um die generelle Knappheit bezahlbaren Wohnraums in Frankfurt löst bei diesen Mieter*innen Zukunftsängste aus:

Manche sagen: „Ich zahle danach, wenn die fertig sind, $850 €$. Dafür kriege ich nicht mal eine Zweizimmerwohnung in Frankfurt, deswegen halte ich die Füße still.“" (Interview 5, Mieter*innen)

Angesichts der Perspektivlosigkeit herrscht bei den betroffenen Haushalten der Eindruck vor, ihre Wohnung um jeden Preis halten zu müssen (vgl. dazu auch Winke 2020). Um die kommenden Mietbelastungen zu kompensieren, suchen die Haushalte deshalb nach neuen Arrangements mit weiteren Einsparungen in ihrer Lebensführung. Der Mangel an Umzugsalternativen schürt gleichzeitig Ängste vor den Konsequenzen einer konfrontativen Haltung gegenüber der Vermieterin und bewirkt, dass Konflikte mit Vonovia vermieden werden.

\subsubsection{Ohnmacht und Pessimismus}

Für andere Mieter*innen steht hingegen das Problem des vernachlässigten baulichen Zustands und der damit einhergehenden öffentlichen Wahrnehmung der Siedlung im Vordergrund. Diese Phänomene weisen eine langjährige Kontinuität auf. Immer wieder haben Mieter*innen erfolglos versucht, vorhandene Mängel gegenüber der Vermietungsgesellschaft zu artikulieren und Instandhaltungen einzufordern. Zum einen werden die umfangreichen Bauarbeiten nun als Chance betrachtet, dass auch tatsächliche Missstände behoben werden (siehe 3.3). Dabei scheint sekundär, dass die ursprünglich geforderte Instandhaltung nun in Gestalt einer umfangreichen Modernisierung auf Kosten der Mieter*innen erfolgt. Zum anderen haben die jahrelange Ignoranz durch Vonovia und die enttäuschten Erwartungen auf Seiten der Mieter*innen Frustration und einen tiefgreifenden Pessimismus bezüglich der Einschätzung ihrer individuellen und kollektiven Handlungsmöglichkeiten erzeugt. Schnell festigt sich so die Ansicht, es handle sich um eine ohnehin nicht abwendbare Entscheidung, auf die im besten Falle noch partiell Einfluss genommen werden kann:

Ich weiß gar nicht, [...] ob das einfach auch nur verschwendete Energie ist, über einen Willen nachzudenken, dass es besser wird. Es ist schwierig, ich glaube 
nicht, dass es irgendwie hier in solchen Siedlungen besser wird, ich kann es mir nicht vorstellen, meine Vorstellungskraft reicht nicht, ich müsste überrascht werden. (Interview 1, Mieterin)

Die von der Vermietungsgesellschaft dauerhaft übergangenen Bedürfnisse erzeugen so paradoxerweise Zustimmung zu den Modernisierungsarbeiten. Vor diesem Hintergrund setzt sich innerhalb der Mieterinitiative zu Beginn eine kooperative Haltung gegenüber der Eigentümerin durch. Ihr zugrunde liegt die Idee einer konstruktiven Zusammenarbeit, über die eine möglichst schnelle und reibungsfreie Verbesserung der konstatierten baulichen Zustände erzielt werden soll. Ein Mieter der Initiative kommentiert, man dürfe ,Vonovia keine Knüppel zwischen die Beine werfen“, sinnvoll sei „nicht die Brechstange, sondern [sich] kooperativ [zu] zeigen“ (Beobachtungsprotokoll 12.06.2019).

Die zunächst paradox erscheinenden Handlungspraktiken werden damit letztlich doch erklärbar. Während individuelle Handlungsstrategien ein kollektives Vorgehen der Initiative behindern, erzeugen spezifische Erfahrungen von Prekarität im Siedlungskontext Frustration, aber auch Zukunftsängste, Pessimismus und Resignation. Diese bewegen viele Mieter*innen - und zu Anfang auch mehrheitlich die Mieterinitiative - zur Akzeptanz der Modernisierung und einer kooperativen Haltung gegenüber der Vermieterin. Somit bestätigt die Studie zentrale Erkenntnisse der Prekaritätsforschung, dass Prekarität zu einer Geringschätzung der individuellen Einflussmöglichkeiten und einer ausbleibenden Bereitschaft $\mathrm{zu}$ widerständigen Praktiken führt. Allerdings kann auch gezeigt werden, dass diese Phänomene nicht essentialistisch oder kulturalistisch als Eigenschaft „,der Prekären“ zu deuten sind, sondern als Konsequenzen der spezifischen materiellen Wohn- und Lebensverhältnisse.

\subsection{Diskursive Deutungsmuster}

Ebenso bietet auch die Analyse verbreiteter Deutungsmuster Erkenntnisse darüber, weshalb sich nicht mehr Widerstand gegen die Modernisierung regt und sich innerhalb der Mieterinitiative nicht früher und in einem stärkeren Maße gemeinschaftliches Handeln und solidarische Vorstellungen gegenüber anderen Mieter*innen etablieren. Hervorzuheben sind diesbezüglich zum einen die territoriale Stigmatisierung (Wacquant 2008) der Siedlung und zum anderen diskriminierende Zuschreibungen und Spaltungen innerhalb der Mieterschaft.

\subsubsection{Territoriale Stigmatisierung der Siedlung}

Viele Mieter*innen empfinden Scham aufgrund der baulichen und optischen Zustände der Siedlung, deren Instandhaltung jahrzehntelang von der Vermietungsgesellschaft vernachlässigt worden ist. So schildert eine Mieterin:

Ich lade keine Freunde oder Familie mehr ein, seitdem ich sowas [Müll, Schrott] auf der Straße sehe. Ich, ich schäme mich. Also als meine Mutter mich äh mich besucht hat und [...] sie hat sich am Geländer verletzt, ja. Weil das alles 
verrostet ist und aufgeplatzt ist und da hat sie nur gesagt, ,in was für einem Drecksloch lebst du hier eigentlich“. Ich sage: „Der Vermieter kümmert sich halt nicht." (Interview 1, Mieterin)

Bewohner*innen berichten von einer negativen und stigmatisierenden öffentlichen Wahrnehmung ihrer Siedlung aufgrund der baulichen Vernachlässigung; stellvertretend steht dafür der Begriff „Ghetto“. In den Gesprächen wird deutlich, dass Mieter*innen diese Wahrnehmung verinnerlichen und den abwertenden Blick selbst reproduzieren. Die mit der Modernisierung verbundene Aussicht, nicht länger als sozial abgehängtes Wohnmilieu wahrgenommen zu werden und sich vom Stigma des schlechten Quartiers befreien zu können, ruft daher bei vielen Mieter*innen Zustimmung und Freude über die Modernisierungsmaßnahmen hervor - selbst bei Haushalten, für welche die Mieterhöhungen eine gravierende finanzielle Belastung bedeuten.

\subsubsection{Diskriminierende Zuschreibungen und Spaltungen innerhalb der Mieterschaft}

Ein weiteres prägnantes Deutungsmuster besteht in der gerade von langjährigen Mieter*innen betriebenen Glorifizierung der ,früheren“ Siedlung. In Kontrast zur aktuellen Situation befeuert diese Verklärung Spaltungen innerhalb der Mieterschaft.

Also früher war es eigentlich gepflegter, sehr Ordnung, also ich weiß, ich bin ja hier gegenüber von den Häusern aufgewachsen, das ist ja eine ganz andere Gesellschaft [...] Heute ist das alles total egal. (Interview 5, Mieter*innen)

Die derzeitige Lage ließe sich - vielen Mieter*innen zufolge - durch ausbleibende Kommunikation und egoistisches Verhalten unter den Nachbar*innen sowie zunehmende Belästigungen (z. B. durch Müll und Lärm) charakterisieren. Dieses Bild entsteht oft auch in Anlehnung an Berichte über die negative Wahrnehmung der Siedlung durch Außenstehende und wird kaum mit der beschriebenen Vernachlässigung der Siedlung durch die Eigentümerin oder den fehlenden sozialen Begegnungsorten in Verbindung gesetzt. Mehrere Gesprächspartner*innen führen die wahrgenommenen negativen Veränderungen vornehmlich auf den verstärkten Zuzug von Nachbar*innen mit Migrationserfahrung zurück. Die Überforderung einiger Mieter*innen mit wahrgenommenen kulturellen Differenzen und insbesondere den Sprachbarrieren stellt ein omnipräsentes Phänomen des Siedlungslebens dar. Während regelmäBiger Kontakt nur zwischen wenigen Mieter*innen besteht, scheint sich für viele der soziale Austausch oftmals auf konflikthafte Konstellationen zu beschränken. Darüber hinaus werden Probleme innerhalb des sozialen Gefüges der Siedlung von manchen Mieter*innen kulturalisiert und damit als mangelnde Anpassungsfähigkeit der Zugezogenen an die hiesige „Kultur“ präsentiert. Dieses essentialistische Kulturverständnis vertieft Spaltungstendenzen zwischen alteingesessenen und neu zugezogen Mieter*innen und befördert die Verbreitung von Schuldzuweisungen und diskriminierenden Zuschreibungen. Die Mischung aus diskriminierenden Stereotypen, Abgrenzungsmechanismen, Überforderung im Umgang miteinander, baulicher Vernachlässigung sowie Ignoranz der Vermietungsgesellschaft hat eine politisch äußerst heikle Konstellation geschaffen. Trotz dieser spaltenden Umstände existiert 
unter den Mieter*innen jedoch zugleich ein starkes Bedürfnis nach mehr Zusammenhalt und Gemeinschaft.

\subsection{Wie in gemeinschaftlicher Praxis dennoch politische Kollektivität entsteht}

Nach mehreren Monaten gemeinsamer Praxis weisen die dominierenden Handlungsperspektiven und Deutungsmuster innerhalb der Mieterinitiative jedoch erhebliche Veränderungen auf. Zum einen wird die Modernisierung selbst nun viel klarer als politischer Aushandlungsprozess betrachtet, zum anderen festigen sich konfrontativere Positionen gegenüber der Vermieterin. Die Mieter*innen haben ein deutlich strategischeres Verständnis ihrer Aufgaben entwickelt, was sich konkret in der Anwendung politischer Druckmittel, wie etwa Pressearbeit, Unterschriftensammlungen, einem gemeinsamen Forderungskatalog und der Vernetzung mit anderen stadtpolitischen Gruppen, ausdrückt. Universellere Forderungen im Sinne aller Mieter*innen setzen sich zunehmend durch, wie etwa die nach einer Deckelung aller Mieten auf maximal 30\% der Nettohaushaltseinkommens. Innerhalb der ursprünglichen Gruppe sind andere Mieter*innen präsenter geworden, wodurch sich die inhaltlichen Positionen der Initiative auch insgesamt verschoben haben. Zudem haben die kontinuierlichen Treffen der Initiative dazu beigetragen, die ursprünglich großen Informationsgefälle zwischen den Anwesenden anzugleichen sowie die organisatorischen Fähigkeiten und das argumentative Selbstbewusstsein einiger Mieter*innen zu stärken. Kleinere Verhandlungserfolge gegenüber Vonovia bestärken die Mitglieder darin, dass sich kollektiver Druck doch bewährt. ${ }^{6}$

In den ersten Monaten nach Gründung der Mieterinitiative in der Siedlung Westhausen haben die ethnographischen Beobachtungen verschiedene Hürden und Mechanismen sichtbar gemacht, welche Spaltungen und Ausschlüsse innerhalb der Mieterschaft sowie die Schwerfälligkeit solidarischer und gemeinschaftlicher Praktiken verstehbar machen (3.1-3.3). Diese frühen Beobachtungen unterstreichen, dass sich aus der Betroffenheit von prekären Wohnverhältnissen nicht automatisch kollektive oder politische Handlungsperspektiven ableiten. Im Gegenteil wurde deutlich, dass vielfältige sozialräumliche Bedingungen, Handlungspraktiken und Deutungsmuster der Entstehung einer politischen Kollektivität unter Mieter*innen erst einmal entgegenstehen. Die spätere Erhebungsphase bringt allerdings bemerkenswerte Dynamiken zum Vorschein, die im Sinne Candeias (2009) aufzeigen, dass Menschen in prekären Wohnverhältnissen nicht per se soziale Vereinzelung und Unorganisierbarkeit unterstellt werden kann. Trotz ihrer starken Prägung durch die sozialstrukturellen Rahmenbedingungen weisen Deutungsmuster und Handlungspraktiken auch unter prekären Verhältnissen Veränderungen sowie ein Potenzial zu politischer Selbstorganisierung auf. Diese Veränderungen lassen sich im Fallbeispiel maßgeblich auf die kontinuierliche Organisierungsarbeit im Rahmen der Mieterinitiative zurückführen, die neue soziale Beziehungen und gemeinschaftliche Praktiken des Austausches etabliert hat. Insgesamt bestätigt dies die Annahme, dass politische Kollektivität erst in vergemeinschafteten Praktiken entstehen kann. Trotz aller Widrigkeiten können

\footnotetext{
${ }^{6}$ Dazu zählt beispielsweise die Offenlegung von abgerechneten Betriebskosten durch Vonovia in Folge einer Unterschriftensammlung.
} 
Organisierungsansätze - in diesem Fall angestoßen und langfristig solidarisch begleitet von externem Fachpersonal der Sozialen Arbeit und eines Mietervereins vermittelt über die konkrete gemeinsame Praxis und anknüpfend an ein diffuses Bedürfnis nach Zusammenhalt, Solidarität und Gemeinschaft also Formen der politischen Kollektivität hervorbringen.

\section{Fazit: Politische Kollektivität durch die Vergemeinschaftung von Perspektiven}

Im Fokus des Beitrages stehen die Wahrnehmung von prekären Wohnverhältnissen durch betroffene Mieter*innen sowie die Bedingungen für die Entstehung politischer Kollektivität, welche am Fallbeispiel der Frankfurter Wohnsiedlung Westhausen ethnographisch untersucht wurden. In Reaktion auf die Ankündigung umfangreicher Modernisierungsarbeiten hat sich bereits zu einem frühen Zeitpunkt auf Anstoß und mit wesentlicher Unterstützung eines Sozialarbeiters sowie einer Mitarbeiterin eines Mietervereins die Mieterinitiative Westhausen gegründet. Diese wird jedoch gerade anfänglich von den meisten Beteiligten nicht als politisches Projekt kollektiven Widerstands betrachtet. Im Vordergrund steht für die Mieter*innen vielmehr der Informationsaustausch und die fachliche Beratung, welche sie für individuelle und kooperativ ausgerichtete Aushandlungsstrategien gegenüber der Vonovia nutzen. Mehrheitlich spricht sich die Initiative für die Akzeptanz der Maßnahmen und eine nicht konflikthafte Haltung gegenüber der Vermieterin aus. Gleichzeitig findet ein Großteil der Haushalte in der Siedlung keinen Anschluss an die Initiative. Als Gründe für das beobachtete Ausbleiben politischer Kollektivität können die sozialstrukturelle Heterogenität der Mieter*innen und die damit einhergehenden divergenten Interessenslagen, Sprachbarrieren, fehlende soziale Begegnungsorte und schwach ausgebildete soziale Netzwerke, Zukunftsängste und Ohnmachtsgefühle als Resultat prekärer Wohnverhältnisse, die Verinnerlichung der territorialen Stigmatisierung sowie diskriminierende Zuschreibungen und Spaltungen innerhalb der Mieterschaft benannt werden. Trotz dieser beträchtlichen Hemmnisse gelingt es der Initiative im zeitlichen Verlauf zunehmend, auch politischere und kollektive Perspektiven herauszubilden.

Die Problematik divergent erfahrener Prekarität innerhalb heterogener Bewohnerschaften und deren Implikationen für das Entstehen politischer Kollektivität greift auch Lisa Vollmer (2019) im Kontext ihrer Untersuchungen zu Mieterinitiativen in Berlin und New York auf. Vollmer verweist im Anschluss an Ernesto Laclau darauf, dass Kollektivität aus äußerst unterschiedlichen Erfahrungen hervorgehen kann, sofern es gelingt, Erlebnisse und Perspektiven zu ,vergemeinschaften“ (Vollmer 2019, S. 125) und von einer individuellen Betroffenheit zu abstrahieren. Nur über den gemeinsamen Austausch kann sich Toleranz und ein Verständnis für anders erfahrene Prekarität ausbilden sowie allmählich ein geteiltes strukturelles und auch politisches Verständnis der erlebten Einschränkungen und Bedrohungen entwickeln. Im Fall der Frankfurter Siedlung Westhausen besteht dies potenziell darin, dass Vonovia trotz kontinuierlicher Mietsteigerungen ihre Instandhaltungspflichten über Jahre verletzt, damit die schlechte Wohnqualität zu verantworten und die Bedürfnis- 
se der Mieter*innen ignoriert hat; nun aber die Modernisierungen zur strategischen Aufwertung ihres Wohnungsbestandes nutzt, deren Kosten dauerhaft auf die Mieter*innen abgewälzt werden können. Ein solches Verständnis verlässt die Ebene individueller Einschränkungen, stärkt kollektives Handeln und schafft als übergreifende Erklärung der Prozesse Anschlussfähigkeit. Das Politische bzw. der Wille, Probleme der Wohnsituation kollektiv und politisch adressieren zu wollen, scheint der Gemeinschaft damit nicht vorgelagert, sondern nachgeschaltet zu sein und nur aus vergemeinschafteten Erfahrungen heraus erwachsen zu können (vgl. auch Vollmer 2019, S. 218).

Die empirischen Ergebnisse der Untersuchung sprechen dafür, dass die beobachteten Hindernisse für die Entstehung politischer Kollektivierung auch als grundlegende Probleme im Westhausener Siedlungsleben zu verstehen sind. Sowohl bei der Frage der politischen Kollektivität als auch bei der Suche nach den Voraussetzungen für nachbarschaftlichen Zusammenhalt - als Gegenentwurf zur sozialen Isolation vieler Mieter*innen innerhalb ihrer Siedlung - erweisen sich allägliche soziale Interaktionen und die Ausbildung sozialer Netzwerke als wichtigste und zugleich schwierige Voraussetzungen. Nur über den Aufbau kontinuierlicher und gemeinschaftlicher Interaktionen können Bedingungen entstehen, dank derer unterschiedlich erfahrene Lebens- und Wohnverhältnisse sowie bestehende Differenzen zwischen den Mieter*innen aufgedeckt, vergemeinschaftet und damit verhandelbar gemacht werden können. Gerade letzterer Punkt ist angesichts der verbreiteten Stigmatisierung als auch der Abgrenzungsversuche innerhalb der Mieterschaft essenziell. Als Konsequenzen für die Praxis städtischer sozialer Bewegungen als auch für Akteure der Sozialen Arbeit lässt sich aus den Ergebnissen ableiten, dass Initiativen politischer Organisierung von Mieter*innen in prekären Wohnverhältnissen häufig einen Anstoß und eine längerfristige Unterstützung von Personen von außen benötigen, die über gewisse Fachkenntnisse, politische Erfahrungen sowie Kompetenzen in den Bereichen Moderation, Organisation, Übersetzung und Öffentlichkeitsarbeit verfügen. Als äußerst nachteilig für die Ausbildung breiter sozialer Netzwerke in der Siedlung Westhausen erweisen sich neben sprachlichen Barrieren vor allem die fehlenden sozialen Infrastrukturen (Gemeindetreff, Cafés, öffentliche Sitzflächen o. Ä.). Möglichkeiten zur sozialen Interaktion mit niedrigschwelligem Zugang existieren abgesehen vom Jugendtreff der Siedlung kaum noch. Der fehlende Dialog zwischen Bewohner*innen kristallisiert sich immer wieder als zentrales Problem heraus und erweist sich daher als wichtiger Anknüpfungspunkt etwa für Akteure der Sozialen Arbeit, um über Begegnungsorte und gemeinschaftlich nutzbare Räume die Vereinzelung aufbrechen und Interaktion auch in heterogenen Nachbarschaften verstärken zu können. Die letzteren beiden Punkte sind damit zugleich notwendige Bedingungen für die Entstehung einer politischen Kollektivität. Eine zentrale Bedeutung scheint in Westhausen zudem der Entwicklung eines gemeinsamen und positiven Ortsverständnisses zuzukommen, um dem stigmatisierenden Blick auf die Siedlung als „Ghetto“ oder der Glorifikation früherer Siedlungszeiten ein inklusives, lebendiges und auf Vielfalt beruhendes Ortsverständnis (Massey 1991) entgegenzusetzen. Auch wenn in der Siedlung kaum übergreifende oder abstrakte Vorstellungen von Solidarität zu finden sind - oftmals eher Differenzen, Konkurrenzen und Spaltungen -, tauchen im Alltag immer wieder kleine solidarische Gesten in Form von Flur- 
gesprächen, gegenseitigen Einladungen oder kleinen Geschenken auf. Neben Frustration und negativ besetzten Vorstellungen werden in fast allen Gesprächen ebenso positiv konnotierte Erfahrungen des aktuellen Siedlungslebens sowie Ideen für die Errichtung und Nutzung neuer gemeinsamer Infrastrukturen angesprochen. Für soziale Bewegungen als auch die Soziale Arbeit im Quartier bieten diese Bedürfnisse nach Zusammenhalt, Solidarität und Gemeinschaft wertvolle Anknüpfungspunkte, die gleichzeitig als Grundlage zur Entstehung einer politischen Kollektivität unter Mieter*innen in prekären Wohnverhältnissen dienen können.

Funding Open Access funding enabled and organized by Projekt DEAL.

Open Access Dieser Artikel wird unter der Creative Commons Namensnennung 4.0 International Lizenz veröffentlicht, welche die Nutzung, Vervielfältigung, Bearbeitung, Verbreitung und Wiedergabe in jeglichem Medium und Format erlaubt, sofern Sie den/die ursprünglichen Autor(en) und die Quelle ordnungsgemäß nennen, einen Link zur Creative Commons Lizenz beifügen und angeben, ob Änderungen vorgenommen wurden.

Die in diesem Artikel enthaltenen Bilder und sonstiges Drittmaterial unterliegen ebenfalls der genannten Creative Commons Lizenz, sofern sich aus der Abbildungslegende nichts anderes ergibt. Sofern das betreffende Material nicht unter der genannten Creative Commons Lizenz steht und die betreffende Handlung nicht nach gesetzlichen Vorschriften erlaubt ist, ist für die oben aufgeführten Weiterverwendungen des Materials die Einwilligung des jeweiligen Rechteinhabers einzuholen.

Weitere Details zur Lizenz entnehmen Sie bitte der Lizenzinformation auf http://creativecommons.org/ licenses/by/4.0/deed.de.

\section{Literatur}

Amt für Wohnungswesen Stadt Frankfurt am Main (2018). Mietspiegel 2018. https://rankfurt.de/themen/ planen-bauen-und-wohnen/wohnen/informationen-zum-wohnungsmarkt/mietspiegel. Zugegriffen: 29. Sept. 2021.

Atkinson, R. (2015). Losing one's place: narratives of neighbourhood change, market injustice and symbolic displacement. Housing, Theory and Society, 32(4), 373-388.

Baldenius, T., Kohl, S., \& Schularick, M. (2020). Die neue Wohnungsfrage. Gewinner und Verlierer des deutschen Immobilienbooms. Leviathan, 48(2), 195-236.

Belina, B. (2018). Wenn Geldkapital eine sichere Bank sucht. Theorie und die politökonomischen Grundlagen der aktuellen Wohnungsfrage in Deutschland. Prokla. Zeitschrift für kritische Sozialwissenschaft, 48(2), 187-203.

Beran, F., \& Nuissl, H. (2019). Verdrängung auf angespannten Wohnungsmärkten: Das Beispiel Berlin. Wüstenrot Stiftung.

Bernien, S. (2017). Verdrängung hat viele Gesichter. In U. Altrock \& R. Kunze (Hrsg.), Stadterneuerung und Armut. Jahrbuch Stadterneuerung, (Bd. 2016, S. 37-63). Springer VS.

Böhnke, P. (2011). Ungleiche Verteilung politischer und zivilgesellschaftlicher Partizipation. Aus Politik und Zeitgeschehen, 1-2/2011, 18-25.

Candeias, M. (2009). Von Anomie zur Organisierung: Die Pariser Banlieu. In R. Castel \& K. Dörre (Hrsg.), Sozialwissenschaften 2009. Prekarität, Abstieg, Ausgrenzung: Die soziale Frage am Beginn des 21. Jahrhunderts (S. 369-379). Campus.

Dörhöfer, K. (1978). Wohnverhältnisse und Bewohnerverhalten. PROKLA. Zeitschrift für kritische Sozialwissenschaft, 8(33), 65-87.

Dörre, K. (2010). Soziale Klassen im Prozess kapitalistischer Landnahmen. In H. Bude (Hrsg.), Marx. Ein toter Hund? Gesellschaftstheorie reloaded (S. 198-236). VSA.

Egner, B., Grohs, S., \& Robischon, T. (Hrsg.). (2021). Stadtforschung aktuell. Die Rückkehr der Wohnungsfrage: Ansätze und Herausforderungen lokaler Politik. Springer VS. 
Ehlers, J. (2021). Ein Erfolgsmodell: Die Nachbarschaftsinitiative Nordend-Bornheim-Ostend (NBO). In J. Betz, S. Keitzel, J. Schardt, S. Schipper, S. P. Schmitt \& F. Wiegand (Hrsg.), Frankfurt am Maineine Stadt für alle? Konfliktfelder, Orte und soziale Kämpfe (S. 363-371). transcript.

Helbrecht, I. (Hrsg.). (2016). Gentrifizierung in Berlin: Verdrängungsprozesse und Bleibestrategien. transcript.

Hölzl, C. (2015). Protestbewegungen und Stadtpolitik: Urbane Konflikte in Santiago de Chile und Buenos Aires. transcript.

Kuge, J., Naumann, M., Nuissl, H., \& Schipper, S. (2020). Angewandte und Kritische Geographie. Gemeinsame Herausforderungen, gemeinsame Perspektiven? Standort. Zeitschrift für Angewandte Geographie, 44(4), 219-225.

Lees, L., Slater, T., \& Wyly, E. K. (2008). Gentrification. Routledge.

Marchart, O. (2014). Die Prekarisierungsgesellschaft. Soziologische Theorie. transcript.

Massey, D. (1991). A global sense of place. Marxism Today, 6, 24-29.

Meuth, M., \& Reutlinger, C. (2021). Von Gentrifizierung betroffen: Ein exemplarischer Beitrag zur Diskussion konzeptioneller und methodisch-methodologischer Fragen qualitativer Verdrängungsforschung. sub urban. zeitschrift für kritische stadtforschung, 9(1/2), 11-30.

Mösgen, A., \& Schipper, S. (2017). Gentrifizierungsprozesse im Frankfurter Ostend. Stadtpolitische Aufwertungsstrategien und Zuzug der Europäischen Zentralbank. Raumforschung und Raumordnung, 75(2), 125-141.

Mullis, D. (2017). Krisenproteste in Athen und Frankfurt: Raumproduktionen der Politik zwischen Hegemonie und Moment. Westfälisches Dampfboot.

Nachtwey, O. (2016). Die Abstiegsgesellschaft: Über das Aufbegehren in der regressiven Moderne. Suhrkamp.

Reutlinger, C., Furrer, H., Hilti, N., Lingg, E., Meuth, M., \& Roth, P. (2019). Perspektive: Wohnungsverlust - Untersuchung zur sozialen Seite baulich-planerischer Strategien. Soziale Passagen, 11(1), 215-219.

Rink, D., \& Vollmer, L. (2019). „Mietenwahnsinn stoppen!“ Netzwerke und Mobilisierungen der Mieter*innenbewegung in deutschen Großstädten. Forschungsjournal Soziale Bewegungen, 32(3), 337-349.

Rink, D., Holm, A., Schönig, B., \& Gardemin, D. (2015). Städte unter Druck. Die Rückkehr der Wohnungsfrage. Blätter für deutsche und internationale Politik, 6, 69-79.

Rutland, T. (2013). Activists in the making: urban movements, political processes and the creation of political subjects. International Journal of Urban and Regional Research, 37(3), 989-1011.

Scheller, D. (2019). Demokratisierung der Postdemokratie: Städtische soziale Bewegungen in Berlin und New York City. transcript.

Schipper, S. (2017). Social movements in an era of post-democracy: how the Israeli J14 tent protests of 2011 challenged neoliberal hegemony through the production of place. Social \& Cultural Geography, 18(6), 808-830.

Schipper, S. (2018). Wohnraum dem Markt entziehen? Wohnungspolitik und städtische soziale Bewegungen in Frankfurt und Tel Aviv. Springer VS.

Schönig, B. (2020). Paradigm shifts in social housing after welfare-state transformation: learning from the German experience. International Journal of Urban and Regional Research, 44(6), 1023-1040.

Schönig, B., \& Vollmer, L. (Hrsg.). (2020). Interdisziplinäre Wohnungsforschung. Wohnungsfragen ohne Ende?! Ressourcen für eine soziale Wohnraumversorgung. transcript.

Slater, T. (2006). The eviction of critical perspectives from gentrification research. International Journal of Urban and Regional Research, 30(4), 737-757.

Steenblock, A., \& Petzold, C. (2021). Tower to the People? Verdrängung durch Modernisierung. Erfahrungen aus dem Brentano-Hochhaus in Frankfurt Rödelheim. In J. Betz, S. Keitzel, J. Schardt, S. Schipper, S.P. Schmitt \& F. Wiegand (Hrsg.), Frankfurt am Main - eine Stadt für alle? Konfliktfelder, Orte und soziale Kämpfe (S. 351-361). transcript.

Thien, H.-G. (2014). Klassentheorien - Die letzten 50 Jahre. PROKLA. Zeitschrift für kritische Sozialwissenschaft, 44(175), 163-190.

Üblacker, J. (2018). Gentrifizierungsforschung in Deutschland: Eine systematische Forschungssynthese der empirischen Befunde zur Aufwertung von Wohngebieten. Budrich.

Unger, K. (2018). Mieterhöhungsmaschinen. Zur Finanzialisierung und Industrialisierung der unternehmerischen Wohnungswirtschaft. PROKLA. Zeitschrift für kritische Sozialwissenschaft, 48(2), 205-225.

Valli, C. (2015). A sense of displacement: long-time residents' feelings of displacement in Gentrifying Bushwick, New York. International Journal of Urban and Regional Research, 39(6), 1191-1208.

Vogelpohl, A., Vollmer, L., Vittu, E., \& Norma, B. (2017). Die Repolitisierung des Wohnens. Städtische soziale Bewegungen für ein Recht auf Wohnen und auf Stadt in Hamburg, Berlin, Jena und Leipzig. 
In B. Schönig, J. Kadi \& S. Schipper (Hrsg.), Wohnraum für alle?! Perspektiven auf Planung, Politik und Architektur (S. 105-130). transcript.

Vollmer, L. (2019). Mieter_innenbewegungen in Berlin und New York: Die Formierung politischer Kollektivität. Springer VS.

Wacquant, L. (2008). Territorial stigmatization in the age of advanced marginality. In J. Houtsonen \& A. Antikainen (Hrsg.), Symbolic power in cultural contexts: uncovering social reality (S. 43-52). Sense Publishers.

Winke, T. (2020). Housing affordability sets us apart: the effect of rising housing prices on relocation behaviour. Urban Studies. https://doi.org/10.1177/0042098020943482. 